\title{
Solvability for fully cantilever beam equations with superlinear nonlinearities
}

Yongxiang $\mathrm{Li}^{1 *}$ and Xuechun Chen'

\section{*Correspondence:} liyxnwnu@163.com

'Department of Mathematics, Northwest Normal University, Lanzhou, People's Republic of China

\section{Abstract}

This paper deals with the existence of solution for the fully fourth-order boundary value problem

$$
\left\{\begin{array}{l}
u^{(4)}(x)=f\left(x, u(x), u^{\prime}(x), u^{\prime \prime}(1), u^{\prime \prime \prime}(x)\right), \quad x \in[0,1] \\
u(0)=u^{\prime}(0)=u^{\prime \prime}(1)=u^{\prime \prime \prime}(1)=0
\end{array}\right.
$$

which models a statically elastic beam fixed at the left and freed at the right, and it is called cantilever beam in mechanics, where $f:[0,1] \times \mathbb{R}^{4} \rightarrow \mathbb{R}$ is continuous. Some inequality conditions on $f$ guaranteeing the existence and uniqueness of solutions are presented. The inequality conditions allow $f\left(x_{1}, y_{0}, y_{1}, y_{2}, y_{3}\right)$ to grow superlinearly on $y_{0}, y_{1}, y_{2}$, and $y_{3}$.

MSC: $34 \mathrm{~B} 15$

Keywords: Cantilever beam equation; Existence and uniqueness; Upperlinear growth; Leray-Schauder fixed point theorem

\section{Introduction and main results}

In this paper we discuss the existence of solution for the fully fourth-order boundary value problem (BVP)

$$
\left\{\begin{array}{l}
u^{(4)}(x)=f\left(x, u(x), u^{\prime}(x), u^{\prime \prime}(x), u^{\prime \prime \prime}(x)\right), \quad x \in[0,1] \\
u(0)=u^{\prime}(0)=u^{\prime \prime}(1)=u^{\prime \prime \prime}(1)=0
\end{array}\right.
$$

where $f:[0,1] \times \mathbb{R}^{4} \rightarrow \mathbb{R}$ is continuous. This problem models deformations of an elastic beam in equilibrium state, whose one end-point is fixed and the other one is freed. In mechanics, the problem is called cantilever beam equation, and in the equation, the physical meaning of the derivatives of the deformation function $u(x)$ is as follows: $u^{(4)}$ is the load density stiffness, $u^{\prime \prime \prime}$ is the shear force stiffness, $u^{\prime \prime}$ is the bending moment stiffness, and $u^{\prime}$ is the slope, see [1-4]. 
For the special case of BVP (1.1) that $f$ does not contain any derivative terms, namely the boundary value problem

$$
\left\{\begin{array}{l}
u^{(4)}(t)=f(x, u(x)), \quad x \in[0,1], \\
u(0)=u^{\prime}(0)=u^{\prime \prime}(1)=u^{\prime \prime \prime}(1)=0,
\end{array}\right.
$$

and $f$ only contains first-order derivative term $u^{\prime}$, namely the boundary value problem

$$
\left\{\begin{array}{l}
u^{(4)}(x)=f\left(t, u(x), u^{\prime}(x)\right), \quad x \in[0,1] \\
u(0)=u^{\prime}(0)=u^{\prime \prime}(1)=u^{\prime \prime \prime}(1)=0
\end{array}\right.
$$

the existence of solutions has been discussed by some authors, see [5-9]. In References [5-7], BVP (1.2) appears as a special case of the $(p, n-p)$ focal boundary value problems for $p=2$ and $n=4$. For the cantilever beam equation with a nonlinear boundary condition of third-order derivative

$$
\left\{\begin{array}{l}
u^{(4)}(x)=f\left(x, u(x), u^{\prime}(x)\right), \quad x \in[0,1], \\
u(0)=u^{\prime}(0)=u^{\prime \prime}(1)=0, \quad u^{\prime \prime \prime}(1)=g(u(1)),
\end{array}\right.
$$

the existence of solution has also been discussed by some authors, see [10-13]. The boundary condition in (1.4) means that the left end of the beam is fixed and the right end of the beam is attached to an elastic bearing device, see [10]. The methods applied in these works are not applicable to BVP (1.1) since they do not deal with the derivative terms $u^{\prime \prime}$ and $u^{\prime \prime \prime}$.

The purpose of this paper is to obtain existence results of solutions to the fully fourthorder BVP (1.1). For fourth-order BVPs with the boundary condition in BVP (1.1) or other boundary conditions, the existence of solutions has been discussed by several authors, see [14-24]. In [14], Kaufmann and Kosmatov considered a symmetric fully fourth-order nonlinear boundary value problem on $[-1,1]$. They used a triple fixed point theorem of cone mapping to obtain existence results of triple positive symmetric solutions under $f$ satisfying some range conditions dependent upon tree positive parameters $a, b$, and $d$. Minhós, Gyulov, and Santos [15] used the method of lower and upper solutions to discuss the existence of a fully fourth-order boundary value problem with a boundary condition different from BVP (1.1) as the discussed problem has a pair of ordered lower and upper solutions. Under the case that $f\left(x, y_{0}, y_{1}, y_{2}, y_{3}\right)$ is linear and sublinear growth on $y_{0}, y_{1}, y_{2}$, $y_{3}$, Li and Liang [16] discussed the existence of the following fully fourth-order boundary value problem:

$$
\left\{\begin{array}{l}
u^{(4)}(x)=f\left(x, u(x), u^{\prime}(x), u^{\prime \prime}(x), u^{\prime \prime \prime}(x)\right), \quad x \in[0,1] \\
u(0)=u(1)=u^{\prime \prime}(0)=u^{\prime \prime}(1)=0
\end{array}\right.
$$

which models a statically bending elastic beam whose two ends are simply supported. In this case, using the method in [16], one can obtain existence results for BVP (1.1). Usually the superlinear problems are more difficult to treat than the sublinear problems. In [17], the author discussed the case that $f\left(x, y_{0}, y_{1}, y_{2}, y_{3}\right)$ may be superlinear growth on $y_{0}, y_{1}$, $y_{2}, y_{3}$, when nonlinearity $f$ is nonnegative by the fixed point index theory in cones. In this 
paper we shall discuss the general case that $f$ may be superlinear growth and have negative value. Our results are as follows.

Theorem 1.1 Assume that $f:[0,1] \times \mathbb{R}^{4} \rightarrow \mathbb{R}$ is continuous and it satisfies the following conditions:

(F1) There exist constants $a_{0}, a_{1}, a_{2}, a_{3} \geq 0$ satisfying $\frac{a_{0}}{8}+\frac{a_{1}}{4}+\frac{a_{2}}{2}+a_{3}<1$ and $b>0$ such that

$$
-f\left(x, y_{0}, y_{1}, y_{2}, y_{3}\right) y_{2} \leq a_{0} y_{0}^{2}+a_{1} y_{1}^{2}+a_{2} y_{2}^{2}+a_{3} y_{3}^{2}+b
$$

for all $\left(x, y_{0}, y_{1}, y_{2}, y_{3}\right) \in[0,1] \times \mathbb{R}^{4}$;

(F2) Given any $M>0$, there is a positive continuous function $\phi_{M}(r)$ defined on $\mathbb{R}^{+}$satisfying

$$
\int_{0}^{+\infty} \frac{r \mathrm{~d} r}{\phi_{M}(r)}=+\infty
$$

such that

$$
\left|f\left(x, y_{0}, y_{1}, y_{2}, y_{3}\right)\right| \leq \phi_{M}\left(\left|y_{3}\right|\right)
$$

for any $x \in[0,1],\left|y_{0}\right|,\left|y_{1}\right|,\left|y_{2}\right| \leq M, y_{3} \in \mathbb{R}$.

Then BVP (1.1) has at least one solution.

In Theorem 1.1, Condition (F1) is easy to be verified and it allows $f\left(x, y_{0}, y_{1}, y_{2}, y_{3}\right)$ be superlinear growth on $y_{0}, y_{1}, y_{2}, y_{3}$. Condition (F2) is a Nagumo-type growth condition on $y_{3}$ which restricts $f$ on $y_{3}$ to be at most quadric growth. This Nagumo-type condition is different from the one (F0) presented in [17], in which (F0) is a Nagumo-type growth condition on $y_{2}$ and $y_{3}$, and (F2) is weaker than (F0). An applied example of Theorem 1.1 will be given at the end of the paper. Strengthening Condition (F1) of Theorem 1.1, we can obtain the following uniqueness result.

Theorem 1.2 Assume that $f:[0,1] \times \mathbb{R}^{4} \rightarrow \mathbb{R}$ is continuous and it satisfies $(F 2)$ and the following condition:

(F3) There exist constants $a_{0}, a_{1}, a_{2}, a_{3} \geq 0$ satisfying $\frac{a_{0}}{8}+\frac{a_{1}}{4}+\frac{a_{2}}{2}+a_{3}<1$ and $b>0$ such that

$$
-\left[f\left(x, y_{0}, y_{1}, y_{2}, y_{3}\right)-f\left(x, z_{0}, z_{1}, z_{2}, z_{3}\right)\right]\left(y_{2}-z_{2}\right) \leq \sum_{i=0}^{3} a_{i}\left(y_{i}-z_{i}\right)^{2}
$$

for all $\left(x, y_{0}, y_{1}, y_{2}, y_{3}\right),\left(x, z_{0}, z_{1}, z_{2}, z_{3}\right) \in[0,1] \times \mathbb{R}^{4}$.

Then BVP (1.1) has a unique solution.

In Condition (F3), by choosing $z_{0}=z_{1}=z_{2}=z_{3}=0$, it follows that (F1) holds, in which $b=\max _{0 \leq x \leq 1}|f(x, 0,0,0,0)|$. Hence Condition (F3) is the strengthening of (F1) and Theorem 1.2 is an improvement of Theorem 1.1. The proof of Theorem 1.1 and Theorem 1.2 is based on Leray-Schauder fixed point theorem and a prior estimate method, which will be given in the next section. 


\section{Proof of the main results}

Let $I=[0,1], C(I)$ denote the Banach space of all continuous functions $u(t)$ on $I$ with norm $\|u\|_{C}=\max _{t \in I}|u(t)|, L^{2}(I)$ be the usual Hilbert space with the inner product $(u, v)=$ $\int_{0}^{1} u(t) v(t) d t$ and the norm $\|u\|_{2}=\left(\int_{0}^{1}|u(t)|^{2} d t\right)^{1 / 2}$. Generally, for $n \in \mathbb{N}, C^{n}(I)$ denotes the Banach space of all $n$ th-order continuous differentiable functions on $I$ with the norm $\|u\|_{C^{n}}=\max \left\{\|u\|_{C},\left\|u^{\prime}\right\|_{C}, \ldots,\left\|u^{(n)}\right\|_{C}\right\}, H^{n}(I)$ is the usual Sobolev space with the norm $\|u\|_{n, 2}=\left(\sum_{i=0}^{n}\left\|u^{(i)}\right\|_{2}^{2}\right)^{1 / 2} \cdot u \in H^{n}(I)$ means that $u \in C^{n-1}(I), u^{(n-1)}(t)$ is absolutely continuous on $I$ and $u^{(n)} \in L^{2}(I)$.

To discuss BVP (1.1), we consider the corresponding linear fourth-order boundary value problem (LBVP)

$$
\left\{\begin{array}{l}
u^{(4)}(x)=h(x), \quad t \in I, \\
u(0)=u^{\prime}(0)=u^{\prime \prime}(1)=u^{\prime \prime \prime}(1)=0
\end{array}\right.
$$

with nonhomogeneous term $h \in L^{2}(I)$.

Lemma 2.1 For every $h \in L^{2}(I), \operatorname{LBVP}(2.1)$ has a unique solution $u:=S h \in H^{4}(I)$, which satisfies

$$
\left\|u^{(i-1)}\right\|_{2} \leq \frac{1}{\sqrt{2}}\left\|u^{(i)}\right\|_{2}, \quad i=1,2,3,4 .
$$

Moreover, the solution operator $S: L^{2}(I) \rightarrow H^{4}(I)$ is a linear bounded operator.

Proof For any given $h \in L^{2}(I)$, it is easy to verify that

$$
u(x)=\int_{0}^{x}(x-s) \int_{s}^{1}(t-s) h(t) \mathrm{d} t \mathrm{~d} s:=\operatorname{Sh}(x), \quad x \in I,
$$

belongs to $H^{4}(I)$ and it is a unique solution of LBVP (2.1). By the boundary condition in $\operatorname{BVP}(2.1)$,

$$
u(x)=\int_{0}^{x} u^{\prime}(t) \mathrm{d} t, \quad x \in I
$$

Hence, by the Hölder inequality,

$$
|u(x)| \leq \int_{0}^{x}\left|u^{\prime}(t)\right| \mathrm{d} t \leq x^{1 / 2}\left(\int_{0}^{x}\left|u^{\prime}(t)\right|^{2} \mathrm{~d} t\right)^{1 / 2} \leq x^{1 / 2}\left\|u^{\prime}\right\|_{2}, \quad x \in I,
$$

so we obtain that $\|u\|_{2} \leq \frac{1}{\sqrt{2}}\left\|u^{\prime}\right\|_{2}$. Similarly, from the equations

$$
\begin{aligned}
& u^{\prime}(x)=\int_{0}^{x} u^{\prime \prime}(t) \mathrm{d} t, \quad x \in I, \\
& u^{\prime \prime}(x)=-\int_{x}^{1} u^{\prime \prime \prime}(t) \mathrm{d} t, \quad x \in I, \\
& u^{\prime \prime \prime}(x)=-\int_{x}^{1} u^{(4)}(t) \mathrm{d} t, \quad x \in I,
\end{aligned}
$$


we can get that $\left\|u^{\prime}\right\|_{2} \leq \frac{1}{\sqrt{2}}\left\|u^{\prime \prime}\right\|_{2},\left\|u^{\prime \prime}\right\|_{2} \leq \frac{1}{\sqrt{2}}\left\|u^{\prime \prime \prime}\right\|_{2},\left\|u^{\prime \prime \prime}\right\|_{2} \leq \frac{1}{\sqrt{2}}\left\|u^{(4)}\right\|_{2}$, respectively. Hence, (2.2) holds. From expression (2.3), we easily see that $S: L^{2}(I) \rightarrow H^{4}(I)$ is a linear bounded operator.

When $h \in C(I), u=\mathrm{Sh} \in C^{4}(I)$ is a classical solution of LBVP (2.1). By the compactness of the Sobolev embedding $H^{4}(I) \hookrightarrow C^{3}(I)$, the solution operator $S: C(I) \rightarrow C^{3}(I)$ is a completely continuous operator.

Let $f:[0,1] \times \mathbb{R}^{4} \rightarrow \mathbb{R}$ be continuous. Define a mapping $F: C^{3}(I) \rightarrow C(I)$ by

$$
F(u)(x):=f\left(x, u(x), u^{\prime}(x), u^{\prime \prime}(x), u^{\prime \prime \prime}(x)\right), \quad x \in I .
$$

By the continuity of $f, F: C^{3}(I) \rightarrow C(I)$ is continuous. Define a composite mapping by

$$
A=S \circ F \text {. }
$$

By the complete continuousness of $S: C(I) \rightarrow C^{3}(I), A: C^{3}(I) \rightarrow C^{3}(I)$ is completely continuous. By the definition of the solution operator $S$ of LBVP (2.1), the solution of BVP (1.1) is equivalent to the fixed point of $A$.

Proof of Theorem 1.1 Let $A: C^{3}(I) \rightarrow C^{3}(I)$ be the completely continuous mapping defined by (2.9). Then the solution of BVP (1.1) is equivalent to the fixed point of $A$. We use the Leray-Schauder fixed point theorem [25] to show that $A$ has a fixed point. For this, we consider the homotopic family of the operator equations

$$
u=\lambda A u, \quad 0<\lambda<1 .
$$

We show that the set of solutions of Eqs. (2.10) is bounded in $C^{3}(I)$.

Let $u \in C^{3}(I)$ be a solution of an equation of (2.10) for $\lambda \in(0,1)$. Set $h=\lambda F(u)$, then $u=\lambda A u=\lambda S(F(u))=S(\lambda F(u))=S h$. By the definition of $S, u=S h$ is the unique solution of LBVP (2.1). Hence $u_{1} \in C^{4}(I)$ satisfies the differential equation

$$
\left\{\begin{array}{l}
u^{(4)}(x)=\lambda f\left(x, u(x), u^{\prime}(x), u^{\prime \prime}(x), u^{\prime \prime \prime}(x)\right), \quad x \in I, \\
u(0)=u^{\prime}(0)=u^{\prime \prime}(1)=u^{\prime \prime \prime}(1)=0 .
\end{array}\right.
$$

Multiplying this equation by $-u^{\prime \prime}(x)$, by Condition (F1), we have

$$
\begin{aligned}
-u^{(4)}(x) u^{\prime \prime}(x) & =-\lambda f\left(x, u(x), u^{\prime}(x), u^{\prime \prime}(x), u^{\prime \prime \prime}(x)\right) u^{\prime \prime}(x) \\
& \leq \lambda\left[a_{0} u^{2}(x)+a_{1} u^{\prime 2}(x)+a_{2} u^{\prime \prime 2}(x)+a_{3} u^{\prime \prime \prime 2}(x)+b\right] \\
& \leq a_{0} u^{2}(x)+a_{1} u^{\prime 2}(x)+a_{2} u^{\prime \prime 2}(x)+a_{3} u^{\prime \prime \prime 2}(x)+b, \quad x \in I .
\end{aligned}
$$

Integrating this inequality on $I$, using integration by parts and the boundary condition of (2.11) for the left side and Lemma 2.1 for the right side, we have

$$
\begin{aligned}
\left\|u^{\prime \prime \prime}\right\|_{2}^{2} & \leq a_{0}\|u\|_{2}^{2}+a_{1}\left\|u^{\prime}\right\|_{2}^{2}+a_{2}\left\|u^{\prime \prime}\right\|_{2}^{2}+a_{3}\left\|u^{\prime \prime \prime}\right\|_{2}^{2}+b \\
& \leq\left(\frac{a_{0}}{8}+\frac{a_{1}}{4}+\frac{a_{2}}{2}+a_{3}\right)\left\|u^{\prime \prime \prime}\right\|_{2}{ }^{2}+b .
\end{aligned}
$$


From this inequality it follows that

$$
\left\|u^{\prime \prime \prime}\right\|_{2}^{2} \leq \frac{b}{1-\left(\frac{a_{0}}{8}+\frac{a_{1}}{4}+\frac{a_{2}}{2}+a_{3}\right)}:=M_{0}
$$

By this inequality and (2.2) of Lemma 2.1, we have

$$
\|u\|_{3,2} \leq\left(\sum_{i=0}^{3}\left\|u^{(i)}\right\|_{2}^{2}\right)^{1 / 2} \leq\left(\frac{1}{8}+\frac{1}{4}+\frac{1}{2}+1\right)^{1 / 2}\left\|u^{\prime \prime \prime}\right\|_{2} \leq 2 M_{0}^{1 / 2}
$$

Hence, by the boundedness of the Sobolev embedding $H^{3}(I) \hookrightarrow C^{2}(I)$,

$$
\|u\|_{C^{2}} \leq C\|u\|_{3,2} \leq 2 C M_{0}^{1 / 2}=: M
$$

where $C$ is the constant of the Sobolev embedding $H^{3}(I) \hookrightarrow C^{2}(I)$.

For this $M>0$, by Condition (F2), there is a positive continuous function $\phi_{M}(r)$ on $\mathbb{R}^{+}$ satisfying (1.6) such that (1.7) holds. By (2.14),

$$
|u(x)|,\left|u^{\prime}(x)\right|,\left|u^{\prime \prime}(x)\right| \leq\|u\|_{C^{2}} \leq M, \quad x \in I .
$$

Hence from (1.7) it follows that

$$
\left|f\left(x, u(x), u^{\prime}(x), u^{\prime \prime}(x), u^{\prime \prime \prime}(x)\right)\right| \leq \phi_{M}\left(\left|u^{\prime \prime \prime}(x)\right|\right), \quad x \in I .
$$

By (1.6), there exists $M_{1}>M$ such that

$$
\int_{0}^{M_{1}} \frac{r \mathrm{~d} r}{\phi_{M}(r)}>2 M
$$

We use (2.15) and (2.16) to show that

$$
\left\|u^{\prime \prime \prime}\right\|_{C} \leq M_{1}
$$

Let $\left\|u^{\prime \prime \prime}\right\|>0$. Since $u^{\prime \prime \prime}(1)=0$, by the maximum theorem of continuous functions, there exists $\xi_{0} \in[0,1)$ such that

$$
\left\|u^{\prime \prime \prime}\right\|_{C}=\max _{x \in I}\left|u^{\prime \prime \prime}(x)\right|=\left|u^{\prime \prime \prime}\left(\xi_{0}\right)\right|
$$

and $u^{\prime \prime \prime}\left(\xi_{0}\right)>0$ or $u^{\prime \prime \prime}\left(\xi_{0}\right)<0$. We only consider the case of that $u^{\prime \prime \prime}\left(\xi_{0}\right)>0$, the other case can be dealt with by a similar method. Set

$$
\xi_{1}=\inf \left\{x \in\left(\xi_{0}, 1\right]: u^{\prime \prime \prime}(x)=0\right\} .
$$

Then, by the continuousness of $u^{\prime \prime \prime}, \xi_{1} \in\left(\xi_{0}, 1\right], u^{\prime \prime \prime}\left(\xi_{1}\right)=0$ and

$$
u^{\prime \prime \prime}(x)>0, \quad x \in\left[\xi_{0}, \xi_{1}\right) .
$$


Hence, by (2.11) and (2.15), we have

$$
\begin{aligned}
-u^{(4)}(x) & =-\lambda f\left(x, u(x), u^{\prime}(x), u^{\prime \prime}(x), u^{\prime \prime \prime}(x)\right) \\
& \leq\left|f\left(x, u(x), u^{\prime}(x), u^{\prime \prime}(x), u^{\prime \prime \prime}(x)\right)\right| \\
& \leq \phi_{M}\left(u^{\prime \prime \prime}(x)\right), \quad x \in\left[\xi_{0}, \xi_{1}\right] .
\end{aligned}
$$

From this it follows that

$$
-\frac{u^{\prime \prime \prime}(x) u^{(4)}(x)}{\phi_{M}\left(u^{\prime \prime \prime}(x)\right)} \leq u^{\prime \prime \prime}(x), \quad x \in\left[\xi_{0}, \xi_{1}\right]
$$

Integrating both sides of this inequality on $\left[\xi_{0}, \xi_{1}\right]$ and making the variable transformation $r=u^{\prime \prime \prime}(x)$ for the left side, we have

$$
\int_{0}^{u^{\prime \prime \prime}\left(\xi_{0}\right)} \frac{r \mathrm{~d} r}{\phi_{M}(r)} \leq u^{\prime \prime}\left(\xi_{1}\right)-u^{\prime \prime}\left(\xi_{0}\right) \leq 2\left\|u^{\prime \prime}\right\|_{C} \leq 2\|u\|_{C^{2}} \leq 2 M
$$

From this inequality and (2.16) it follows that $u^{\prime \prime \prime}\left(\xi_{0}\right) \leq M_{1}$. Hence by (2.18), $\left\|u^{\prime \prime \prime}\right\|_{C}=$ $u^{\prime \prime \prime}\left(\xi_{0}\right) \leq M_{1}$, namely (2.17) holds.

Now from (2.14) and (2.17), we conclude that

$$
\|u\|_{C^{3}}=\max \left\{\|u\|_{C^{2}},\left\|u^{\prime \prime \prime}\right\|_{C}\right\} \leq M_{1} .
$$

This means that the set of the solutions of Eqs. (2.10) is bounded in $C^{3}(I)$. By the LeraySchauder fixed point theorem [18], $A$ has a fixed point in $C^{3}(I)$, which is a solution of BVP (1.1).

The proof of Theorem 1.1 is completed.

Proof of Theorem 1.2 Let $b=\max \{|f(x, 0,0,0,0)|: x \in I\}+1$. In Condition (F3), choosing $z_{0}=z_{1}=z_{2}=z_{3}=0$, we conclude that (F1) holds. Hence, by Theorem 1.1, BVP (1.1) has at least one solution.

Let $u_{1}, u_{2} \in C^{4}(I)$ be two solutions of BVP (1.1). Set $u=u_{2}-u_{1}$ and $h=F\left(u_{2}\right)-F\left(u_{1}\right)$. Then $u=u_{2}-u_{1}=A u_{2}-A u_{2}=S\left(F\left(u_{2}\right)\right)-S\left(F\left(u_{2}\right)\right)=$ Sh. Hence $u$ is a solution of LBVP (2.1), and it satisfies the equation

$$
u^{(4)}(x)=F\left(u_{2}\right)(x)-F\left(u_{1}\right)(x), \quad x \in I
$$

Multiplying this equation by $-u^{\prime \prime}(x)=-\left(u_{2}{ }^{\prime \prime}(x)-u_{1}{ }^{\prime \prime}(x)\right)$, by Condition (F3) we obtain that

$$
\begin{aligned}
-u^{(4)}(x) u^{\prime \prime}(x) & =-\left[F\left(u_{2}\right)(x)-F\left(u_{1}\right)(x)\right]\left(u_{2}^{\prime \prime}(x)-u_{1}{ }^{\prime \prime}(x)\right) \\
& \leq \sum_{i=0}^{3} a_{i}\left(u_{2}^{(i)}(x)-u_{1}^{(i)}(x)\right)^{2} \\
& =\sum_{i=0}^{3} a_{i}\left(u^{(i)}(x)\right)^{2}, \quad x \in I .
\end{aligned}
$$


Integrating this inequality on $I$ and using Lemma 2.1, we obtain that

$$
\begin{aligned}
\left\|u^{\prime \prime \prime}\right\|_{2}^{2} & \leq a_{0}\|u\|_{2}^{2}+a_{1}\left\|u^{\prime}\right\|_{2}^{2}+a_{2}\left\|u^{\prime \prime}\right\|_{2}^{2}+a_{3}\left\|u^{\prime \prime \prime}\right\|_{2}^{2} \\
& \leq\left(\frac{a_{0}}{8}+\frac{a_{1}}{4}+\frac{a_{2}}{2}+a_{3}\right)\left\|u^{\prime \prime \prime}\right\|_{2}^{2} .
\end{aligned}
$$

Since $\frac{a_{0}}{8}+\frac{a_{1}}{4}+\frac{a_{2}}{2}+a_{3}<1$, this inequality implies that $\left\|u^{\prime \prime \prime}\right\|_{2}=0$. By $(2.2),\|u\|_{2} \leq \frac{1}{2 \sqrt{2}}\left\|u^{\prime \prime \prime}\right\|_{2}$, so we have $\|u\|_{2}=0$. Hence $u_{1}=u_{2}$. This means that BVP (1.1) has only one solution.

The proof of Theorem 1.2 is completed.

Example 2.1 Consider the following superlinear fourth-order boundary value problem:

$$
\left\{\begin{array}{l}
u^{(4)}=2 u+3 u^{\prime 2} u^{\prime \prime}+5 u^{\prime \prime 3} u^{\prime \prime \prime 2}+x \sin \pi x, \quad x \in I \\
u(0)=u^{\prime}(0)=u^{\prime \prime}(1)=u^{\prime \prime \prime}(1)=0
\end{array}\right.
$$

We verify that the corresponding nonlinearity

$$
f\left(x, y_{0}, y_{1}, y_{2}, y_{3}\right)=2 y_{0}+3 y_{1}^{2} y_{2}+5 y_{2}^{3} y_{3}^{2}+x \sin \pi x
$$

satisfies the conditions of Theorem 1.1. Choose $a_{0}=1, a_{1}=0, a_{2}=\frac{5}{4}, a_{3}=0$, and $b=1$, then $\frac{a_{0}}{8}+\frac{a_{1}}{4}+\frac{a_{2}}{2}+a_{3}=\frac{3}{4}<1$. For every $\left(x, y_{0}, y_{1}, y_{2}, y_{3}\right) \in[0,1] \times \mathbb{R}^{4}$, we have

$$
\begin{aligned}
-f\left(x, y_{0}, y_{1}, y_{2}, y_{3}\right) y_{2} & =-2 y_{0} y_{2}-3 y_{1}^{2} y_{2}^{2}-5 y_{2}{ }^{4} y_{3}{ }^{2}+(x \sin \pi x) y_{2} \\
& \leq 2\left|y_{0}\right|\left|y_{2}\right|+\left|y_{2}\right| \leq y_{0}^{2}+y_{2}{ }^{2}+\frac{1}{4} y_{2}^{2}+1 \\
& =a_{0} y_{0}^{2}+a_{1} y_{1}^{2}+a_{2} y_{2}^{2}+a_{3} y_{3}^{2}+b .
\end{aligned}
$$

Hence, $f$ satisfies Condition (F1). Since $f\left(x, y_{0}, y_{1}, y_{2}, y_{3}\right)$ is quadratic growth on $y_{2}$ by (2.26), it follows that (F2) holds. Hence, by Theorem 1.1, BVP (2.25) has at least one solution. It should be pointed out that this conclusion cannot be obtained from the known results of References [1-17].

Acknowledgements

Not applicable.

Funding

The work is supported by NNSFs of China $(11661071,11761063)$.

\section{Abbreviations}

Not applicable.

Availability of data and materials

Data sharing not applicable to this article as no datasets were generated or analyzed during the current study.

Competing interests

The authors declare that they have no competing interests.

Authors' contributions

$Y L$ and XC carried out the first draft of this manuscript, YL prepared the final version of the manuscript. All authors read and approved the final version of the manuscript. 


\section{Publisher's Note}

Springer Nature remains neutral with regard to jurisdictional claims in published maps and institutional affiliations.

Received: 2 February 2019 Accepted: 22 April 2019 Published online: 29 April 2019

\section{References}

1. Aftabizadeh, A.R.: Existence and uniqueness theorems for fourth-order boundary value problems. J. Math. Anal. Appl. $116,415-426(1986)$

2. Agarwal, R.P.: Boundary Value Problems for Higher Order Differential Equations. World Scientific, Singapore (1986)

3. Gupta, C.P.: Existence and uniqueness theorems for a bending of an elastic beam equation. Appl. Anal. 26, 289-304 (1988)

4. Gupta, C.P.: Existence and uniqueness results for the bending of an elastic beam equation at resonance. J. Math. Anal. Appl. 135, 208-225 (1988)

5. Agarwal, R.P.: Multiplicity results for singular conjugate, focal and (n, p) problems. J. Differ. Equ. 170, 142-156 (2001)

6. Agarwal, R.P., O'Regan, D.: Twin solutions to singular boundary value problems. Proc. Am. Math. Soc. 128, 2085-2094 (2000)

7. Agarwal, R.P., O'Regan, D., Lakshmikantham, V.: Singular $(p, n-p)$ focal and $(n, p)$ higher order boundary value problems. Nonlinear Anal. 42, 215-228 (2000)

8. Yao, Q.: Monotonically iterative method of nonlinear cantilever beam equations. Appl. Math. Comput. 205, 432-437 (2008)

9. Yao, Q.: Local existence of multiple positive solutions to a singular cantilever beam equation. J. Math. Anal. Appl. 363, 138-154 (2010)

10. Ma, T.F., da Silva, J.: Iterative solutions for a beam equation with nonlinear boundary conditions of third order. Appl. Math. Comput. 159, 11-18 (2004)

11. Infante, G., Pietramala, P.: A cantilever equation with nonlinear boundary conditions. Electron. J. Qual. Theory Differ. Equ. 2009, 15 (2009) Special Edition I, 14 pp.

12. Alves, E., Ma, T.F., Pelicer, M.L.: Monotone positive solutions for a fourth order equation with nonlinear boundary conditions. Nonlinear Anal. 71, 3834-3841 (2009)

13. Cabada, A., Tersian, S.: Multiplicity of solutions of a two point boundary value problem for a fourth-order equation. Appl. Math. Comput. 219, 5261-5267 (2013)

14. Kaufmann, E.R., Kosmatov, N.: Elastic beam problem with higher order derivatives. Nonlinear Anal., Real World Appl. 8, $811-821(2007)$

15. Minhos, F., Gyulov, T., Santos, A.I.: Lower and upper solutions for a fully nonlinear beam equation. Nonlinear Anal. 71, 281-292 (2009)

16. Li, Y., Liang, Q.: Existence results for a fully fourth-order boundary value problem. J. Funct. Spaces 2013, Article ID $641617(2013)$

17. Li, Y.: Existence of positive solutions for the cantilever beam equations with fully nonlinear terms. Nonlinear Anal., Real World Appl. 27, 221-237 (2016)

18. Bai, Z:: Positive solutions of some nonlocal fourth-order boundary value problem. Appl. Math. Comput. 215 4191-4197 (2010)

19. Cui, Y., Sun, J.: Existence of multiple positive solutions for fourth-order boundary value problems in Banach spaces Bound. Value Probl. 2012, 107 (2012)

20. Wei, Y., Song, Q., Bai, Z.: Existence and iterative method for some fourth order nonlinear boundary value problems Appl. Math. Lett. 87, 101-107 (2019)

21. Zou, Y.: On the existence of positive solutions for a fourth-order boundary value problem. J. Funct. Spaces 2017, Article ID 4946198 (2017)

22. Jiang, R., Zhai, C.: Positive solutions for a system of fourth-order differential equations with integral boundary conditions and two parameters. Nonlinear Anal., Model. Control 23(3), 401-422 (2018)

23. Benham, A., Kosmatov, N.: Multiple positive solutions of a fourth-order boundary value problem. Mediterr. J. Math. 14 78 (2017)

24. Ma, Y., Yin, C., Zhang, G.: Positive solutions of fourth-order problems with dependence on all derivatives in nonlinearity under Stieltjes integral boundary conditions. Bound. Value Probl. 2019, 41 (2019)

25. Deimling, K.: Nonlinear Functional Analysis. Springer, New York (1985)

\section{Submit your manuscript to a SpringerOpen ${ }^{\circ}$ journal and benefit from:}

- Convenient online submission

- Rigorous peer review

- Open access: articles freely available online

- High visibility within the field

- Retaining the copyright to your article

Submit your next manuscript at $\boldsymbol{~ s p r i n g e r o p e n . c o m ~}$ 